\title{
Control of Roof Plate Development and Signaling by Lmxlb in the Caudal Vertebrate CNS
}

\author{
Victor V. Chizhikov and Kathleen J. Millen \\ Department of Human Genetics, University of Chicago, Chicago, Illinois 60637
}

\begin{abstract}
Numerous studies have identified the roof plate as an important signaling center controlling dorsal interneuron specification and differentiation in the developing spinal cord. Currently, the molecular pathways of roof plate formation and function are poorly understood. We determined that the LIM-homeodomain transcription factor Lmxlb is sufficient to induce functional roof plate in the early chick developing spinal cord. In the chick, Lmxlb acts upstream of Lmxla in the roof plate developmental program. Once the roof plate forms, we show that Bmp and Wnt signaling are the major components of $L m x 1 a / b$-dependent roof plate dorsal patterning activity. The roof plate function of $L m x 1 b$ is not conserved across vertebrates because $L m x 1 b$ is not expressed in mouse roof plate progenitors. Instead, mouse caudal CNS roof plate formation relies entirely on $L m x 1 a$. $L m x 1 b$ can, however, partially rescue roof plate development in dreher $\left(\operatorname{Lm} x 1 a^{-/-}\right)$mice, indicating that $\operatorname{Lmx} 1 \mathrm{~b}$ has some functional redundancy to Lmxla. Furthermore, we demonstrate that the roof plateinducing activity of Lmx1b can be suppressed by Mash1 (Cash1), which is normally expressed in intermediate neural tube in both chick and mouse. Our data identify $L m x l b$ as a key regulator of spinal cord roof plate induction and function.
\end{abstract}

Key words: Lmxlb; roof plate; Lmxla; dreher; Bmp; Wnt; developing spinal cord

\section{Introduction}

The roof plate of the developing CNS is a transient non-neural dorsal midline structure that controls the specification, differentiation, and axonal trajectories of the neurons at the dorsal neural tube (Lee and Jessell, 1999; Butler and Dodd, 2003). In caudal $\mathrm{CNS}$, the roof plate originates from mitotically active progenitors of the neural folds in response to Bmp signaling from the adjacent epidermal ectoderm (Liem et al., 1997; Lee and Jessell, 1999). As development proceeds, roof plate progenitors exit the cell cycle, acquire typical roof plate morphology, and initiate expression of differentiated roof plate markers, including MafB. In addition, the roof plate secretes peptides of the Bmp and Wnt families, which have been proposed to play major roles in the specification of adjacent dorsal interneurons both in vitro and in vivo (Liem et al., 1997; Lee and Jessell, 1999; Muroyama et al., 2002; Timmer et al., 2002; Helms and Johnson, 2003). Although numerous studies have firmly established the importance of the roof plate as a signaling center controlling dorsal CNS patterning, little is understood regarding the molecular pathways driving roof plate induction or function.

\footnotetext{
Received March 10, 2004; revised April 16, 2004; accepted May 12, 2004.

This work was supported by a Seed Grant from the Brain Research Foundation and a grant-in-aid from the Whitehall Foundation (to K.J.M.). We thank K. Sharma for his generous assistance with chick electroporation; R. Johnson, A. McMahon, M. German, T. Jessell, J. Briscoe, J. Timmer, L. Niswander, J. Johnson, D. Anderson, K. Kishimoto, T. Muller, C. Birchmeier, P. Brickell, M. Takeichi, E. Turner, and M. Bronner-Fraser for $L m \times 1 b^{-1-}$ mice, expression constructs, in situ probes, or antibodies; and A. Lindgren, I. Grinberg, and E. Steshina for technical assistance and/or valuable comments on this manuscript.

Correspondence should be addressed to Kathleen J. Millen, Department of Human Genetics, University of Chicago, 920 East 58th Street, Cummings Life Sciences Center 319, Chicago, IL 60637. E-mail: kmillen@genetics.bsd.uchicago.edu.

D0I:10.1523/JNEUROSCI.0758-04.2004

Copyright $\odot 2004$ Society for Neuroscience $\quad 0270-6474 / 04 / 245694-10 \$ 15.00 / 0$
}

We established previously that the LIM-homeodomain protein Lmxla is required for roof plate formation. Lmxla is expressed in the neural folds and in the developing roof plate (Millonig et al., 2000; Failli et al., 2002). Loss of Lmxla function in dreher mutant mice leads to the failure of roof plate formation in the caudal CNS and subsequent disruption of dorsal neural patterning (Millonig et al., 2000). Lmxla is a member of a large family of LIM-homeobox-encoding genes. Within this large, diverse family, Lmxla is most highly related to Lmxlb, a protein with $61 \%$ overall amino acid identity (Hobert and Westphal, 2000).

$L m \times 1 b$ is widely expressed during embryogenesis, and its function is required for normal limb, kidney, eye, and skull development (Riddle et al., 1995; Vogel et al., 1995; Chen et al., 1998; Dreyer et al., 1998). In the CNS, Lmx1b is expressed in the isthmus, developing serotonergic and dopaminergic neurons of the hindbrain and midbrain, and a subset of dorsal interneurons of the spinal cord. Lmxlb is also expressed in the roof plate and floor plate of the neural tube (Yuan and Schoenwolf, 1999; Adams et al., 2000; Smidt et al., 2000; Gross et al., 2002; Matsunaga et al., 2002; Muller et al., 2002; Ding et al., 2003). Although the role of Lmx1b in the isthmus (Adams et al., 2000; Matsunaga et al., 2002) and serotonergic and dopaminergic neurons (Smidt et al., 2000; Ding et al., 2003) is clearly established, its function in dorsal spinal cord development is unclear.

Using chick in ovo electroporation technology in conjunction with mutant mouse analysis, we determined that roof plate induction is the primary activity of Lmxlb in the early developing dorsal spinal cord. $L m x 1 b$ expression in the developing roof plate is activated by Bmp expression, and its roof plate-inducing activity can be inhibited by Mash1 (Cash1), normally expressed in intermediate neural tube. Strikingly, Lmxlb is not expressed in 
the roof plate of the mouse caudal CNS. Mouse spinal cord roof plate development is entirely dependent on Lmxla. Lmx1b acts upstream of Lmxla in the chick roof plate differentiation program and can partially rescue roof plate development in the dreher mouse. Using both gain-of-function and loss-of-function approaches, we identified Bmp signaling as the major component of Lmxla/b-dependent roof plate signaling in chick developing spinal cord. Lmxla/b also activates dorsal midline Wnt 1 expression. The role of Lmxla/b-dependent Wntl expression, however, is limited, regulating the numbers of only the most dorsal neuronal population, the dI1 interneurons.

\section{Materials and Methods}

Embryos. Fertilized White Leghorn eggs were incubated and staged according to Hamburger and Hamilton (1951). dreher $\left(\operatorname{Lmx} 1 a^{-/-}\right)$and $L m \times 1 b$ mutant mice were maintained and genotyped as described previously (Chen et al., 1998; Millonig et al., 2000).

Chick embryo manipulations. In ovo electroporation was used to express proteins of interest in chick developing spinal cord as described previously (Megason and McMahon, 2002). Full-length mouse Lmx1b was cloned upstream of internal ribosomal entry site followed by enhanced green fluorescent protein (IRES-EGFP) in the pCIG expression vector (Megason and McMahon, 2002). Chick Lmxlb was expressed using chick Lmxlb/RCAS(A) virus (Riddle et al., 1995). Mouse Lmxla, Bmp4, Wnt1, dominant-negative (dn) Wnt1, chick Noggin, and Xenopus Follistatin were expressed from pCIG-Lmxla (Chizhikov and Millen, 2004), pMiwII-BMP4 (Kishimoto et al., 2002), pCIG-Wnt1 (Megason and McMahon, 2002), pC1-neo-dnWn1 (Garcia-Castro et al., 2002), pMT-Noggin, and pCMV-Follistatin (Liem et al., 2000), respectively. In all experiments, an appropriate empty vector was used as a control. In cell cycle experiments, $150 \mu \mathrm{l}$ of different concentrations of olomoucin or aphidicolin (Sigma, St. Louis, MO) were applied on top of embryos in ovo $5 \mathrm{hr}$ after electroporaion (h.a.e.), corresponding to the approximate initial expression of the exogenous $d n W n t 1$. Chick intermediate explants from electroporated and control neural plates were isolated according to Liem et al. (1995) and cultured in serum-free medium as described previously (Garcia-Castro et al., 2002).

Mouse embryo manipulations. Embryonic day 9.25 (E9.25) whole dreher embryos or their subdissected neural tubes (Lee et al., 1998) were embedded into collagen and electroporated as described previously (Akamatsu et al., 1999). They were then cultured for $24 \mathrm{hr}$ using previously published conditions (Lee et al., 1998). All cultured embryos and some neural tube explants were processed for immunohistochemistry. Some neural tube explants were used for RNA isolation and reverse transcription (RT)-PCR analysis.

Immunohistochemistry, in situ hybridization, bromodeoxyuridine, and terminal deoxynucleotidyl transferase-mediated biotinylated UTP nick end labeling. Immunohistochemistry was performed on frozen sections as described previously (Helms and Johnson, 1998). The following primary antibodies were used: anti-MafB (Pouponnot et al. 1995), anti-LH2A/B (Lee et al., 1998), anti-Math1 (Helms and Johnson, 1998), anti-Lbx1 (Muller et al., 2002), anti-Lmxla (M. German, unpublished observation; a gift from M. German, University of California San Francisco, San Francisco, CA), anti-Brn3a (Fedtsova and Turner, 1997), anti-Lmx1b, antiIslet1 (51.4H9), anti-Lim1/2 (4F2), anti-Msx1/2 (4G1), anti-Pax7, and anti-Pax6 [all obtained from the Developmental Studies Hybridoma Bank (University of Iowa, Iowa City, IA), developed under the auspices of the National Institute of Child Health and Human Development]. Secondary species-appropriate antibodies with Texas Red or FITC conjugates were obtained from Jackson ImmunoResearch (West Grove, PA). In situ hybridization was performed as described previously (Timmer et al., 2002) using digoxigenin-labeled riboprobes to chick Gdf7, Bmp4, Wnt1, Cash1, and mouse Lmx1b provided by T. Jessell (Columbia University, New York, NY) (Gdf7), P. Brickell (Institute of Child Health, London, UK) (Bmp4), A. McMahon (Harvard University, Cambridge, MA) (Wnt1), J. Johnson (University of Texas Southwestern Medical Center, Dallas, TX) and D. Anderson (California Institute of Technology, Pasadena, CA) (Cash1), and R. Johnson (University of Texas, M.D.
Anderson Cancer Center, Houston, TX) (Lmxlb). Bromodeoxyuridine (BrdU) and terminal deoxynucleotidyl transferase-mediated biotinylated UTP nick end labeling were performed as described previously (Megason and McMahon, 2002). Sections were digitally photographed with an AxioCam on a Zeiss (Oberkochen, Germany) AxioPlan 2 microscope and processed using Adobe Photoshop (Adobe Systems, San Jose, CA).

$R T$-PCR. Each explant was collected into $200 \mu \mathrm{l}$ of extraction buffer, and RNA was extracted using PicoPure RNA extraction kit (Arcturus, Mountain View, CA). Total RNA was reverse transcribed by use of random primers. cDNA was amplified by PCR for 30 cycles. Primer pairs to amplify mouse transcripts were $5^{\prime}$-TCACTGCACGTGGACTTTAAGGAG-3' and 5'-TGATGGGACTGAGCCTTGCG-3' for Gdf7, and 5' ACTGCCGTCGCCGTCGCCATTCACTA-3' and 5'-CACCACCTTGTCATACTCATCCAG-3' for Bmp4. Amplification of a fragment of GAPDH was also performed as a control with primers $5^{\prime}$-TGACGTGCCGCCTGGAGAAA- $3^{\prime}$ and 5'-GGTCCACCACCCTGTTGCTGTA$3^{\prime}$. PCR products were fractionated in $2 \%$ agarose gels and visualized by staining with ethidium bromide.

Cell count and statistical analysis. All sections were taken from the region between forelimbs and hindlimbs. Only sections with sufficient levels of GFP expression (10-15 representative sections per embryo) were used for analysis. All results were replicated in at least four embryos or explants. For quantification of roof plate cells, the total number of positive cells was counted on each section. For quantification of dorsal interneurons, only positive cells on the electroporated side were counted on each section. These numbers were compared with numbers of cells of interest in GFP-electroporated control embryos and not to the numbers of on the non-electroporated slides of the experimental embryos, because we sometimes observed that Lmxlb-induced roof plate nonautonomously affected not only the electroporated but also the control side of the neural tube. All quantitative data are expressed as the mean \pm SEM. Statistical significance was determined by two-tailed $t$ test. ${ }^{*}$ indicates $p<0.01$ in all figures.

\section{Results \\ Roof plate induction by Lmx1b in chick developing dorsal spinal cord}

In the chick developing dorsal spinal cord, $L m x 1 b$ is expressed in the dorsal midline, and dorsal dI5 and dILB interneurons (Fig. $1 A-F$ ) (Yuan and Schoenwolf, 1999; Gross et al., 2002; Muller et al., 2002). In the chick caudal CNS, we first detected Lmx1b expression at the beginning of neurulation, specifically in the elevating and converging neural folds (Fig. $1 A$ ). Just before neural tube closure, expression of the roof plate marker Lmxla was then initiated. In both the neural folds and dorsal midline of the formed neural tube at later stages, the Lmx1b expression completely overlapped with the Lmxla expression domain (as detected by double immunolabeling) (Fig. $1 B, C$ ). Lmx1b expression also completely overlapped with MafB, a specific marker of differentiated roof plate that is first expressed at stage 15 (Fig. $1 D)$. By electroporating stage 10 chick neural plates with a Bmp4 expression vector, we showed that, similar to other roof plate markers (MafB, Bmp4, Wnt1), roof plate $L m x 1 b$ expression is positively regulated by Bmp signaling (data not shown) (Liem et al., 1997; Liu et al., 2004). Strikingly, $L m x 1 b$ expression was not detected in the neural folds or in the dorsal midline of the developing spinal cord in the mouse at any stage investigated (Fig. $1 G$ ), although, in the mouse, $L m \times 1 b$ is clearly expressed in the dI5 and dILB dorsal interneurons (Fig. $1 G$ and data not shown) (Gross et al., 2002; Muller et al., 2002).

Because Lmxlb is highly expressed in roof plate progenitors and differentiated roof plate cells in the chick developing spinal cord, we investigated whether $L m \times 1 b$ could induce roof plate fate. We overexpressed chick and mouse $L m \times 1 b$ in caudal neural plate shortly before neural tube closure using in ovo electroporation of 
chick embryos at stage 10, the time of initiation of endogenous Lmxlb expression in the neural folds. In all experiments described below, overexpression of chick and mouse $L m \times 1 b$ resulted in identical phenotypes (all figures show overexpression of chick $\operatorname{Lm} \times 1 b)$.

Roof plate marker analysis of embryos electroporated with $L m \times 1 b$ revealed an approximate fourfold increase in the number of dorsal MafB-positive cells compared with embryos electroporated with EGFP alone (Fig. 2A-E). In addition, the expression domains of secreted roof plate markers Gdf7, Bmp4, and Wnt1 were also ectopically expanded in embryos electroporated with Lmx1b (10 of 14 embryos) but not EGFP alone ( $n=6$ embryos) (Fig. $2 F-I$ and data not shown). The ectopic expression of all roof plate markers was limited to the most dorsal region of the neural tube, despite widespread electroporation of exogenous Lmx1b $(n=14)$ (Fig. $2 A, B$ and data not shown), suggesting a regionally restricted competence to produce roof plate cells in response to $L m x 1 b$ activity. An alternative hypothesis is that $L m x 1 b$ can only expand already existing roof plate and is not sufficient to induce it de novo. To distinguish these two possibilities, in vitro explant experiments were performed. Control stage 10 intermediate neural tube naive explants $(n=7)$ (Liem et al., 1997) or those electroporated with EGFP alone $(n=5)$ failed to generate any roof plate cells, as assessed by Pax7/MafB double staining (Fig. $2 L-N)$. After $30 \mathrm{hr}$ in culture, however, these control explants initiated coexpression of endogenous Lmxlb and Lbx1, the generic marker of dI4-dI6 interneurons (data not shown). Because in developing spinal cord this marker combination is unique to dI5 dorsal interneurons (Gross et al., 2002; Muller et al., 2002), we conclude that some explants cells adopted dI5 interneuron fate in the absence of dorsalizing or ventralizing signals. In contrast, intermediate explants electroporated with $L m x 1 b$ produced numerous roof plate cells ( $n=4$ explants) (Fig. $2 J, K, N)$, indicating that Lmxlb can induce roof plate de novo. No expression of Lbxl was detected in these explants at any time investigated (data not shown). Thus, $L m x 1 b$ can induce roof plate de novo in stage 10 naive intermediate neural plate explants and can either directly or indirectly override the dI 5 cell fate when it is expressed at this early stage.

Because endogenous roof plate consists of non-dividing differentiated cells, we investigated whether $L m x 1 b$ expression was sufficient to withdraw neural progenitors from the cell cycle. Surprisingly, many cells expressing exogenous $L m x 1 b$ were still BrdU positive as late as 48 h.a.e., and no differences in numbers of BrdU-incorporating cells were detected between Lmx1belectroporated and control sides of the neural tubes (supplemental Fig. 1, available at www.jneurosci.org). Additionally, no changes in BrdU incorporation were detected when $L m x 1 b$ was overexpressed in subdissected E9.25 mouse neural tubes in vitro (data not shown). Thus, although $L m \times 1 b$ can induce expression of all roof plate markers investigated, we found no evidence that $L m \times 1 b$ alters the cell cycle of electroporated cells.
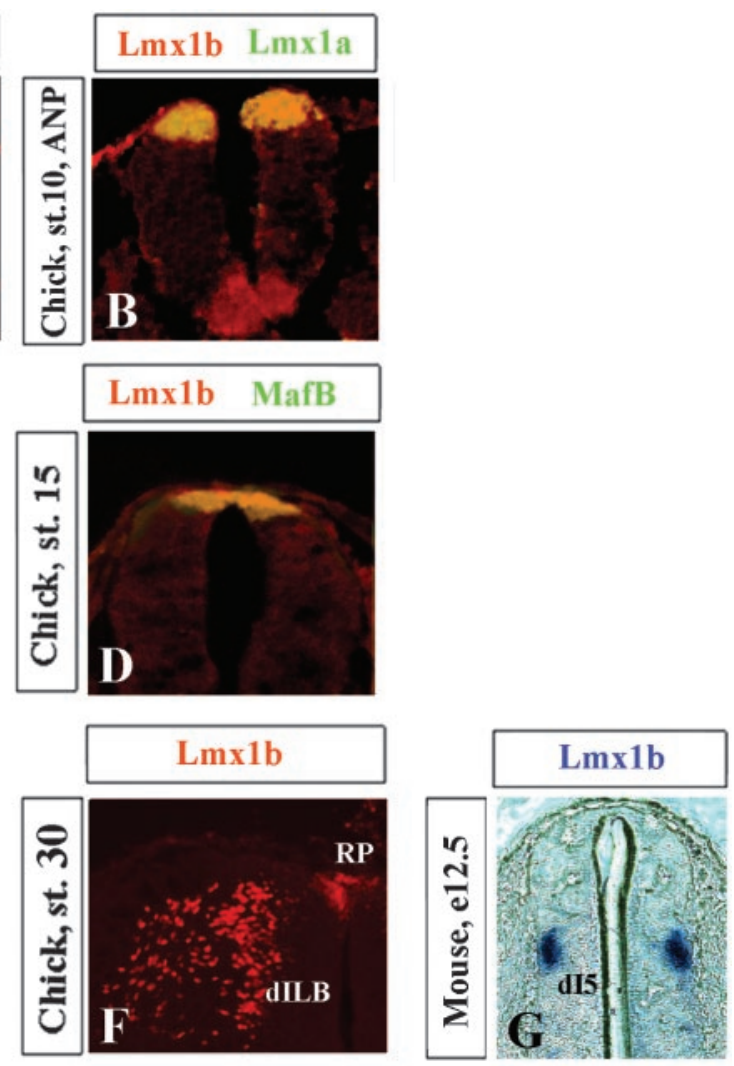

RP

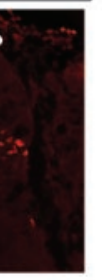

Figure 1. Expression of $L m x 1 b$ in chick and mouse developing spinal cord. Immunofluorescence labeling of $L m x 1 b$ (red) alone $(E, F)$ or together with Lmx1a (green) $(A-C)$, and MafB (green) $(D)$ in chick developing spinal cord at the stages (st.) indicated. PNP, Posterior neural plate; ANP, anterior neural plate. Cells that coexpress two markers appearyellow. Expression of $L m x 1 b$ (blue) detected by in situ hybridization in E12.5 mouse developing spinal cord. RP, Roof plate.

\section{Functional activity of Lmx1b-induced roof plate}

The primary function of roof plate in developing spinal cord is to pattern adjacent dorsal tissue (Lee and Jessell, 1999). To investigate whether the ectopic roof plate induced by exogenous $L m \times 1 b$ in vivo was functional, we first examined the expression of Pax and Msx family members as early indicators of regional identity in developing spinal cord (Liem et al., 1995; Ericson et al., 1997; Timmer et al., 2002). Additionally, we assessed the patterning of $\mathrm{dI} 1, \mathrm{dI} 2, \mathrm{dI} 3$, and $\mathrm{dI} 4-\mathrm{dI} 6$ dorsal spinal cord interneurons (Fig. 3Q). These were identified by their expression of the LIM-homeodomain proteins LH2A/B, Lim1/2, Islet1, and Lbx1, respectively (Gross et al., 2002; Muller et al., 2002) (Fig. 3). To avoid the complications of any potential cellautonomous effects of Lmx1b on dorsal interneuron cell fate, we limited our analysis to sections in which exogenous Lmx1b expression was restricted to only the most dorsal regions of the neural tube (as detected by GFP fluorescence and confirmed by anti-GFP antibody staining) (Fig. $3 B, D, F, I, L, O$ and data not shown).

In unelectroporated embryos, Pax7 and Msx1/2 expression was restricted to dorsal cells of the neural tube (Liem et al., 1995; Timmer et al., 2002). Pax6 was expressed at high levels in the intermediate regions of the neural tube and at reduced levels dorsally (Ericson et al., 1997; Timmer et al., 2002). In the neural tubes of $L m \times 1 b$-electroporated embryos, however, the expression domains of Pax7 and Msx1/2 were significantly extended ventrally (Fig. $3 A, B$ and data not shown). The Pax6 expression domain was reduced and also shifted ventrally (Fig. $3 C, D$ ). There was an approximate fourfold increase in numbers of dI1 inter- 

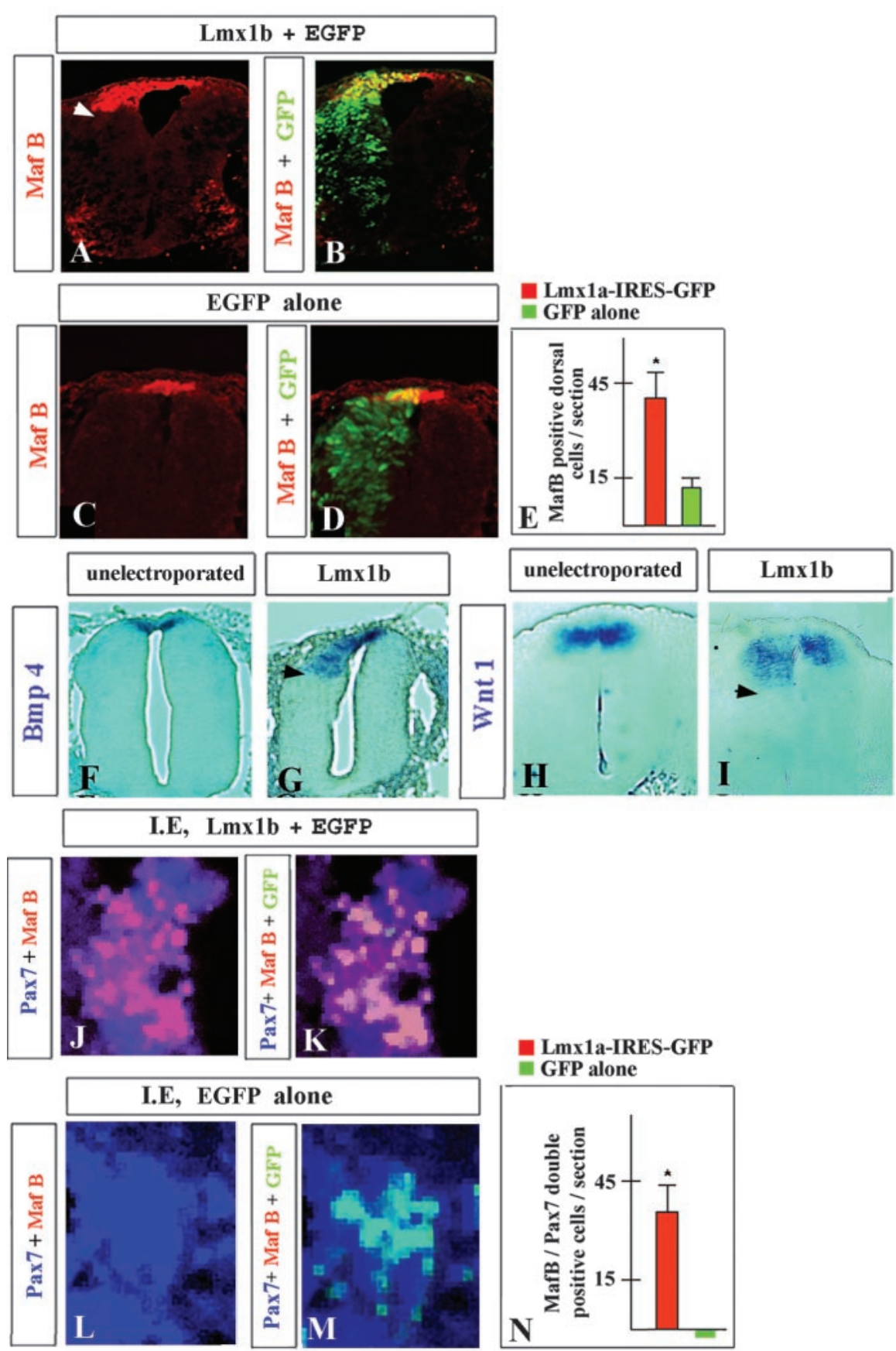

Figure 2. Lmx1b induces roof plate in chick developing spinal cord. Expression of MafB $(A-D)$ (red, detected by immunofluorescence), $B m p 4(F, G)$, and $W n t 1(H, I)$ (both blue, detected by in situ hybridization) in neural tubes of unelectroporated embryos $(F, H)$ and embryos electroporated with either $\operatorname{Lm} x 1 b-\operatorname{EGFP}(A, B, G, I)$ or EGFP alone $(C, D)$. All pictures are from stage 24 embryos, 48 h.a.e. Arrowheads point to the ventral boundary of ectopic domains of expression of roof plate markers induced by exogenous $L m x 1 b$. E, Quantitative analysis of dorsal MafB-positive cells in embryos electroporated with $L m \times 1 b$ and EGFP alone. J-M, Expression of MafB (red) and Pax7 (blue) $(J, L)$ or MafB and Pax7 together with GFP (green) $(K, M)$ and quantitative analysis of roof plate cells $(N)$ in chick intermediate neural plate explants (I.E.) electroporated with $L m \times 1 b-$ $\operatorname{EGFP}(J, K)$ or EGFP alone $(L, M)$.

neurons in samples expressing exogenous $\operatorname{Lmx} 1 b$. Additionally, there was a marked shift of these interneurons to more ventral regions of the neural tube (eight of nine embryos), areas normally populated by dI2 and dI3 interneurons (Fig. 3E-G). No dI2, dI3, or dI4-dI6 interneurons were detected in neural tubes with high levels of exogenous $L m \times 1 b$ expression in the dorsal midline region (Fig. $3 H-P$ ). We conclude that the ectopic roof plate in- duced by exogenous $L m x 1 b$ is functional because it repatterns the regional identity of the developing neural tube (summarized in Fig. 3Q), non-autonomously altering dorsal neuronal specification.

\section{Lmx1b acts upstream of Lmx1a in the} chick roof plate differentiation program and can partially rescue roof plate development in dreher mouse embryos $L m x 1 b$ can induce Lmxla expression when ectopically expressed in both developing spinal cord in vivo (five of seven embryos) (Fig. 4A-C) and in chick naive intermediate neural plate explants in vitro $(40 \pm 7$ Lmxla-positive cells per section; five of six explants) (data not shown). In contrast, Lmxla could not induce Lmx1b expression either in vivo ( $n=5$ embryos) (Fig. $4 D-F)$ or in vitro ( $n=4$ explants) (data not shown), indicating that Lmxlb acts upstream of Lmxla in the roof plate differentiation program. We determined previously that $L m x 1 a$ is sufficient to induce the entire roof plate differentiation program in chick developing spinal cord (our published observations). Thus, the induction of the roof plate by $L m x 1 b$ may be explained by the activation of $L m x l a$ expression. To determine whether the $L m \times 1 b$ roof plate-inducing activity relies entirely on Lmxla, we performed mouse electroporation experiments. We electroporated

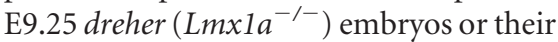
subdissected neural tubes with either chick or mouse $L m x 1 b$-expressing or control vectors, cultured them in vitro for $24 \mathrm{hr}$, and then stained them with antibodies or processed for gene expression analysis by RT-PCR. As expected, unelectroporated dreher embryos or those electroporated with EGFP did not express MafB (Fig. $4 H, J, K), G d f 7$, or $B m p 4$, as detected by RT-PCR (Fig. 4L). In contrast, dreher embryos electroporated with either chick or mouse Lmxlb had numerous MafBpositive cells in the dorsal midline domain of their developing spinal cord (Fig. 4I$K)$. Strong Gdf7 and Bmp4 expression was detected in their neural tubes (Fig. $4 L$ ). These data indicate that, although $L m \times 1 b$ is not expressed in mouse caudal roof plate, both chick and mouse $L m x 1 b$ can at least partially rescue roof plate development in dreher mouse embryos. These assays, however, cannot distinguish between the possibility that Lmx1b has similar functional capacity to Lmxla or whether Lmxlb can induce roof plate via an independent pathway.

The molecular nature of Lmx1-dependent roof plate signaling Bmps and Wnts have been implicated in the development of several classes of dorsal interneurons (Liem et al., 1997; Muroyama et al., 2002; Timmer et al., 2002; Liu et al., 2004), al- 
though many details of their specific roles remain unclear. Because $L m x 1 a / b$ can induce functional roof plate, manipulation of Lmxla/b expression provides an opportunity to investigate the molecular nature of Lmx1-dependent roof plate signaling. We first established that overexpression of Bmp4 in stage 15 chick caudal neural tubes resulted in a phenotype that was indistinguishable from the $L m x 1 b$ overexpression phenotype (compare supplemental Fig. 2, available at www.jneurosci.org, with Fig. 3), suggesting that Bmp signaling likely represents a major component of Lmxlbdependent roof plate signaling. In contrast, ectopic expression of Wnt1 had a very limited effect on dorsal spinal cord development, affecting only dI1 interneuron development (supplemental Fig. 2, available at www.jneurosci.org). Wnt1 overexpression led to a threefold increase in dI1 population (supplemental Fig. $2 H, J$, available at www.jneurosci.org). However, unlike the phenotype caused by $L m x 1 b$ overexpression, these dI 1 interneurons were still located in their endogenous domain and not significantly expanded ventrally (supplemental Fig. $2 \mathrm{H}$, available at www.jneurosci.org). Although previous studies have found that Wntl has a mitogenic effect (Megason and McMahon, 2002), the observed increase in dI1 numbers in Wnt1-electroporated embryos cannot be completely explained by the mitogenic activity of Wnt1, because the numbers of other dorsal interneurons (such as $\mathrm{dI} 2$, dI 3 , and dI4-dI6) in Wnt1-electroporated neural tubes were only moderately increased (supplemental Fig. 2 I, J, available at www.jneurosci.org, and data not shown).

Next, we used an inhibitor approach to directly test the role of Bmp and Wnt signaling as components of Lmxla/b-dependent roof plate signaling. Coelectroporation of $L m \times 1 b$ or Lmx1a with either the Bmp inhibitors noggin and follistatin (Liem et al., 1997) or the Wnt inhibitor $d n W n t 1$ (Garcia-Castro et al., 2002) did not affect the ability of $L m x 1$ genes to induce MafBpositive cells both in vivo and in intermediate neural plate explants in vitro (data not shown). These data indicate that the Bmps and Wnts are not required downstream of Lmxla/b to form roof plate. In contrast, however, Bmp signaling from the $L m x 1 a / b$-induced roof plate is required for roof plate function, because coelectroporation of Lmxla/b together with noggin and follistatin blocked the ability of Lmx1 genes to cause ventral expansion of Pax7 and Msx1/2 expression domains. Instead, these electroporations caused a decrease in Pax7 and Msx1/2 expression in dorsal neural tube and a dorsal shift in the Pax6 expression domain (Fig. 5A,B and data not shown). Coelectroporation of $\operatorname{Lmx} 1 \mathrm{a} / \mathrm{b}$ with noggin and follistatin also blocked the Lmx1-dependent loss of dI4-dI6 interneurons and, instead, expanded their domain dorsally (Fig. 5D,F and data not shown). Additionally, the Bmp inhibitors prevented the ventral extension of dI1 interneurons (Fig. 5C) that was always observed when $L m \times 1 b$ or $L m \times 1 a$ was overexpressed alone. Importantly, however, a electroporation.
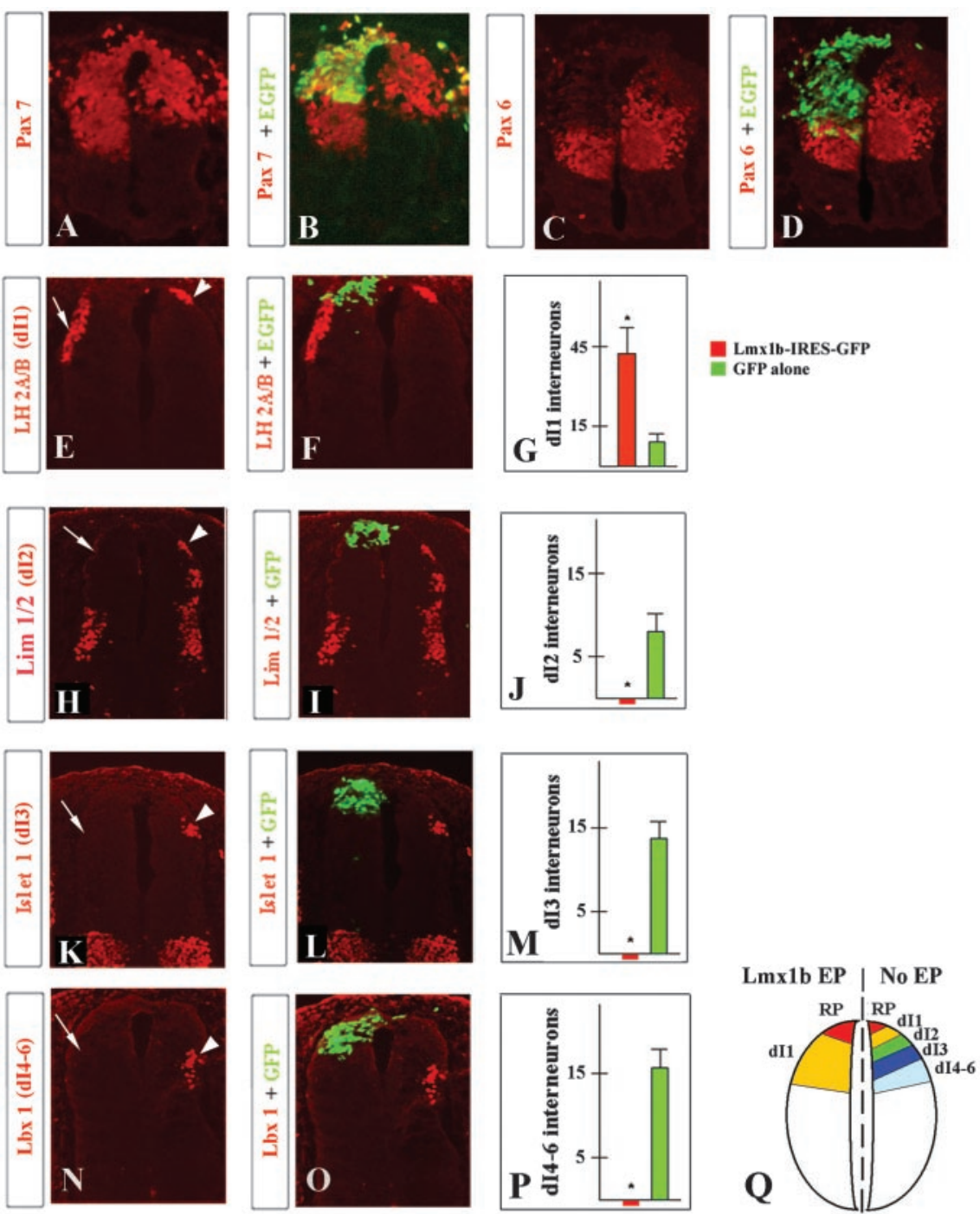

Figure 3. Effects of ectopic $L m x 1 b$-generated roof plate on dorsal and intermediate spinal cord specification. Expression of $\operatorname{Pax} 7(A, B), \operatorname{Pax} 6(C, D), \mathrm{LH} 2 A / B(E, F), \operatorname{Lim} 1 / 2(H, I)$, Islet1 $(K, L)$, and Lbx1 $(N, 0)$ in neural tubes of chick embryos electroporated showing location of dorsal interneurons in non-electroporated and $L m x 1 b$-electroporated chick spinal cord. RP, Roof plate; EP,

slight increase in dI1 interneuron numbers within their endogenous domain was still observed (Fig. 5C,E). These data indicate that Bmps are required to allow $\mathrm{Lmxla/b}$-induced roof plate to repattern adjacent neural tube tissue in our overexpression assays. These data also suggest that there are other Lmx1-dependent factors that specifically positively regulate $\mathrm{dI} 1$ interneuron development, even when Bmp signaling is downregulated.

In contrast to Bmp inhibition, most $L m \times 1 a / b$-overexpression phenotypes were unaffected when either gene was coelectroporated with the Wnt inhibitor $d n W n t 1$. The ventral extension of Pax7 and Msx1/2 expression domain and strong ventral shift of Pax6 expression were still observed (Fig. 5G,H and data not shown). Additionally, no dI4-dI6 interneurons were detected on the electroporated sides of the neural tubes (Fig. $5 \mathrm{~J}, \mathrm{~L}$ and data not shown). Downregulation of Wnt signaling when $L m x 1 a / b$ was overexpressed, however, had a very specific effect on the development of dI1 interneurons. Although dI1 interneurons 
chick, 24 h.a.e.

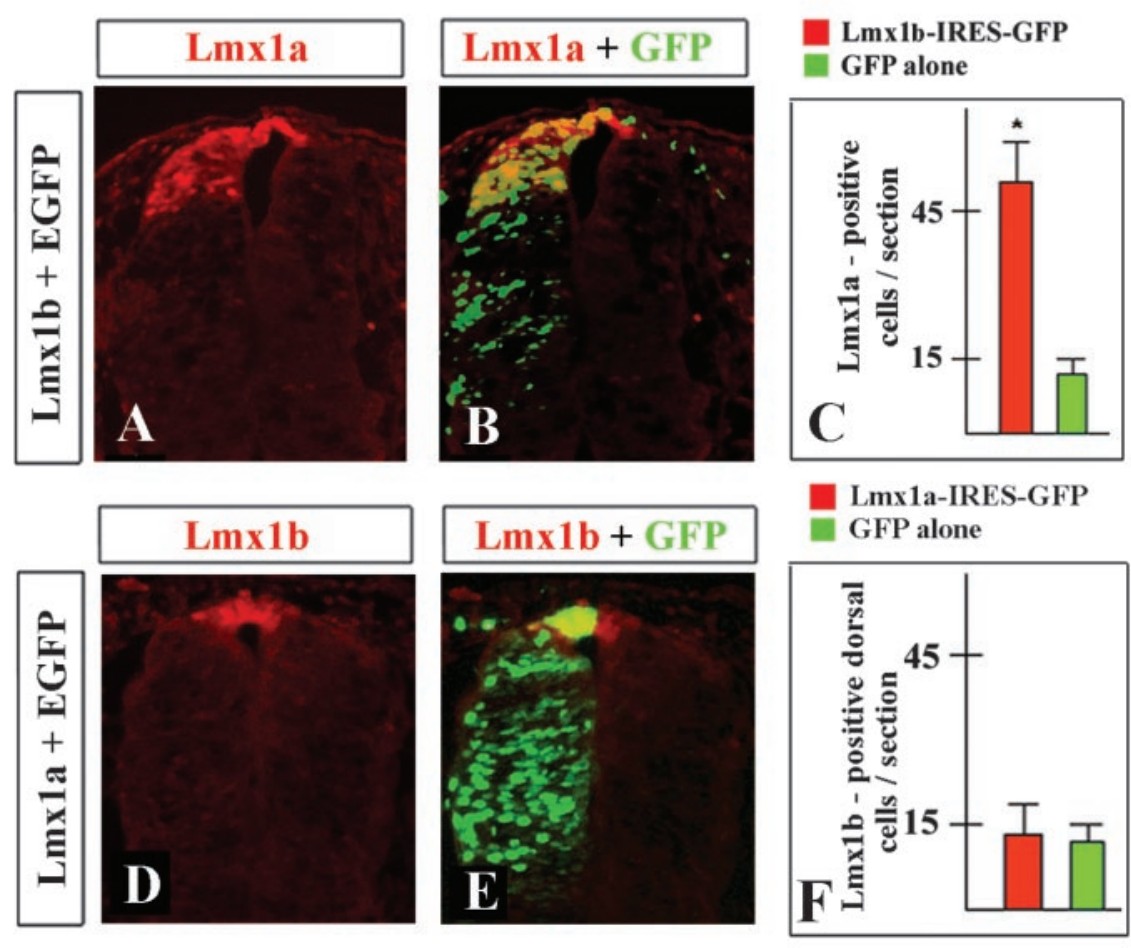

dreher mouse, 24 h.a.e.
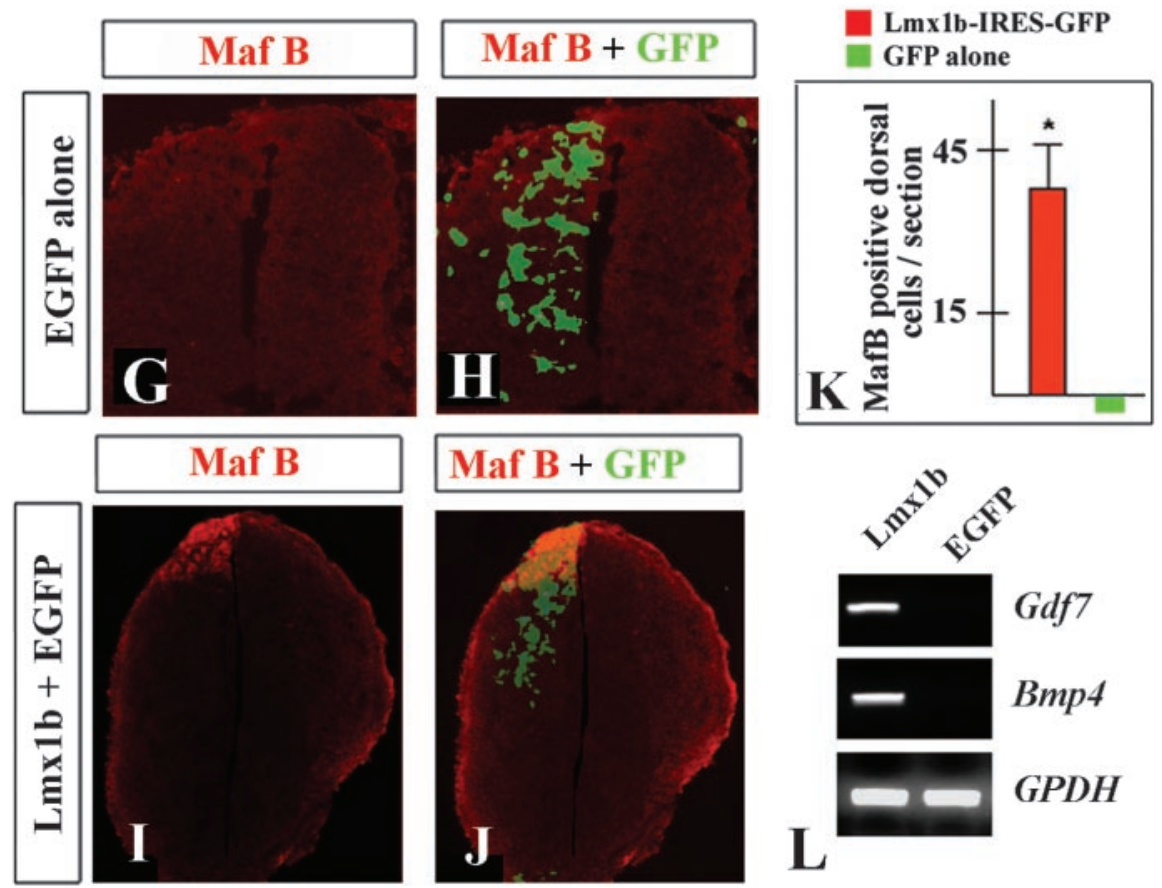

Figure 4. $\quad$ Lmx1b acts upstream of $\operatorname{Lmx1a}$ and can partially rescue roof plate phenotype in dreher mouse embryos. $A-C$, Expression of $\operatorname{Lmx} 1 \mathrm{a}(A, B)$ and quantitative analysis of numbers of $\operatorname{Lmx} 1$ a-positive cells $(C)$ in chick neural tubes electroporated with $\operatorname{Lmx} 1 \mathrm{~b} 24$ h.a.e. $D-F$, Expression of $\operatorname{Lmx} 1 \mathrm{~b}(D, E)$ and quantitative analysis of numbers of $L m x 1 b$-positive cells $(F)$ in chick neural tubes electroporated with $L m x 1 a 24$ h.a.e. $G-K$, Expression of MafB $(G-J)$ and quantitative analysis of number of MafB-positive cells $(K)$ in developing spinal cords of dreher mouse embryos electroporated with $L m x 1 b(I, J)$ or EGFP alone $(G, H) 24$ h.a.e. $L, R T-P C R$ analysis of expression of $G d f 7$ and Bmp4 in dreher developing spinal cords electroporated with $L m \times 1 b$ and EGFP. were significantly expanded ventrally in neural tubes coelectroporated with $L m \times 1 b$ and $d n W n t 1$, their increase in number was only moderate (25\% increase) (Fig. $5 I, K)$. This increase was much lower than that in neural tubes expressing exogenous $L m \times 1 b$ alone (fourfold increase), indicating that downregulation of Wnt signaling partially suppressed the activity of $L m x 1 a / b$ to induce dI1 interneurons. Because Wnts have been reported previously to be mitogenic signals for dorsal neural tube cells (Megason and McMahon, 2002), it was possible that the negative effect of dnWntl on Lmxla/b-dependent dI1 interneuron induction is attributable to an inhibition of cellular proliferation. Alternatively, Wnt signaling may regulate Lmxl-dependent dI1 interneuron specification more directly. To distinguish these possibilities, we evaluated the effects of dnWntl on the proliferation of dorsal neural tube cells. When the $L m \times 1 b$ and $d n W n t 1$ expression vectors ( $1 \mu \mathrm{g} / \mu \mathrm{l}$ each) were coelectroporated, we usually observed a $70 \%$ decrease of BrdU incorporation in dorsal neural tubes compared with overexpression of $L m \times 1 b$ alone. We duplicated this $70 \%$ reduction of BrdU incorporation by applying the cell cycle inhibitors olomoucin $(200 \mu \mathrm{M})$ or aphidicolin $(10 \mu \mathrm{M})$ on the top of Lmx1b (1 $\mu \mathrm{g} / \mu \mathrm{l})$-electroporated chick embryos (data not shown). Despite the presence of these inhibitors, $L m \times 1 b$ overexpression still caused a twofold increase in the number of dI1 interneurons (Fig. $5 K$ ). Thus, a decrease of cell proliferation cannot fully account for the entire decrease in the number of Lmxlb-induced dI1 interneurons when Wnt signaling is downregulated. Instead, we conclude that Wnt1 has a more specific role in Lmxla/bdependent dI1 interneuron specification. To determine whether other signaling pathways have discernable roles in roof plate function, we coelectroporated $L m \times 1 b$ or Lmxla together with noggin, follistatin, and $d n W n t 1$. No dI1 interneurons were detected in the majority of sections of these embryos. We therefore conclude that, in our overexpression assays, Bmps and Wnts act as the major components of Lmx1dependent roof plate signaling. Bmp signaling influences the specification of numerous dorsal cellular populations, whereas Wnt signaling specifically regulates dI1 specification.

Lmx1b is not involved in dI5 and dILB interneuron specification

$L m \times 1 b$ is expressed not only in roof plate but also in other cellular populations of the spinal cord, including dI5 and dILB 
interneurons in both chick and mouse (Fig. $1 E-G$ ). To test whether $L m x 1 b$ is involved in specification and early differentiation of these neurons, we electroporated $L m \times 1 b$ into stage 15 chick neural tubes, at the time of initiation of normal exogenous Lmx Ib dI5 expression. To avoid the complications of any non-autonomous effects of ectopic Lmxlb-induced roof plate on dorsal interneuron cell fate, we limited our analysis to sections in which the expression of exogenous $L m x 1 b$ was restricted to intermediate regions of the neural tube (supplemental Fig. $3 B, D$, available at www.neurosci.org, and data not shown). Using LH2, Isl1, Lim1/2, Brn3a, and Lbx1 immunostaining, we found that $L m x 1 b$ overexpression neither induced nor repressed expression of any of these markers (supplemental Fig. $3 A-D$, available at www.jneurosci.org, and data not shown). dI5 and dILB cell fates were also assessed in $L m \times 1 b$ targeted null mutant mice (Chen et al., 1998). Using an $L m x 1 b$ in situ probe that detected both wild-type and mutant transcripts, we found no difference between wild-type and $L m \times 1 b^{-/-}$embryos in either the number or distribution of dorsal $L m x 1 b$ positive cells at E11.5 or E12.5 (data not shown). Immunohistochemical analysis revealed that dI5 and dILB neurons (and flanking dI1-dI4 and dI6 interneurons) were normally specified in $L m \times 1 b^{-/-}$embryos (supplemental Fig. $3 E-H$, available at www.jneurosci.org, and data not shown). Furthermore, there was no difference in the numbers of proliferating and apoptotic cells in dorsal and intermediate developing spinal cord of wild-type and $L m \times 1 b^{-/-}$embryos at E10.5-E12.5. (data not shown). We conclude that $L m \times 1 b$ has no role in the specification or early differentiation of dI5 and dILB interneurons.

Mash1, normally expressed in intermediate neural tube progenitors, can suppress roof plate-inducing activity of Lmx1b

Our data indicate that, because overexpression of $L m \times 1 b$ in stage 10 naive intermediate neural plate explants induces roof plate and prevents expression of dI5 interneuron markers, roof plate induction is the primary role of $L m x 1 b$ in the early developing chick spinal cord. At later stages, however, dI5 and dILB interneurons express endogenous $L m \times 1 b$ in both chick and mouse without acquiring roof plate properties. This suggests that, during normal development, the roof plate-inducing activity of Lmxlb must become suppressed in intermediate cells and limited to the dorsal midline. Evidence of repression is also provided by our electroporation of $L m x 1 b$ into chick stage 10 neural plates. In these experiments, ectopic $L m \times 1 b$-induced roof plate was always limited to the most dorsal region of the neural tube and was never observed in intermediate spinal cord (Fig. $2 A, B$ and data not shown). One olomoucin.

\section{Lmx1b + noggin + follistatin electroporated}
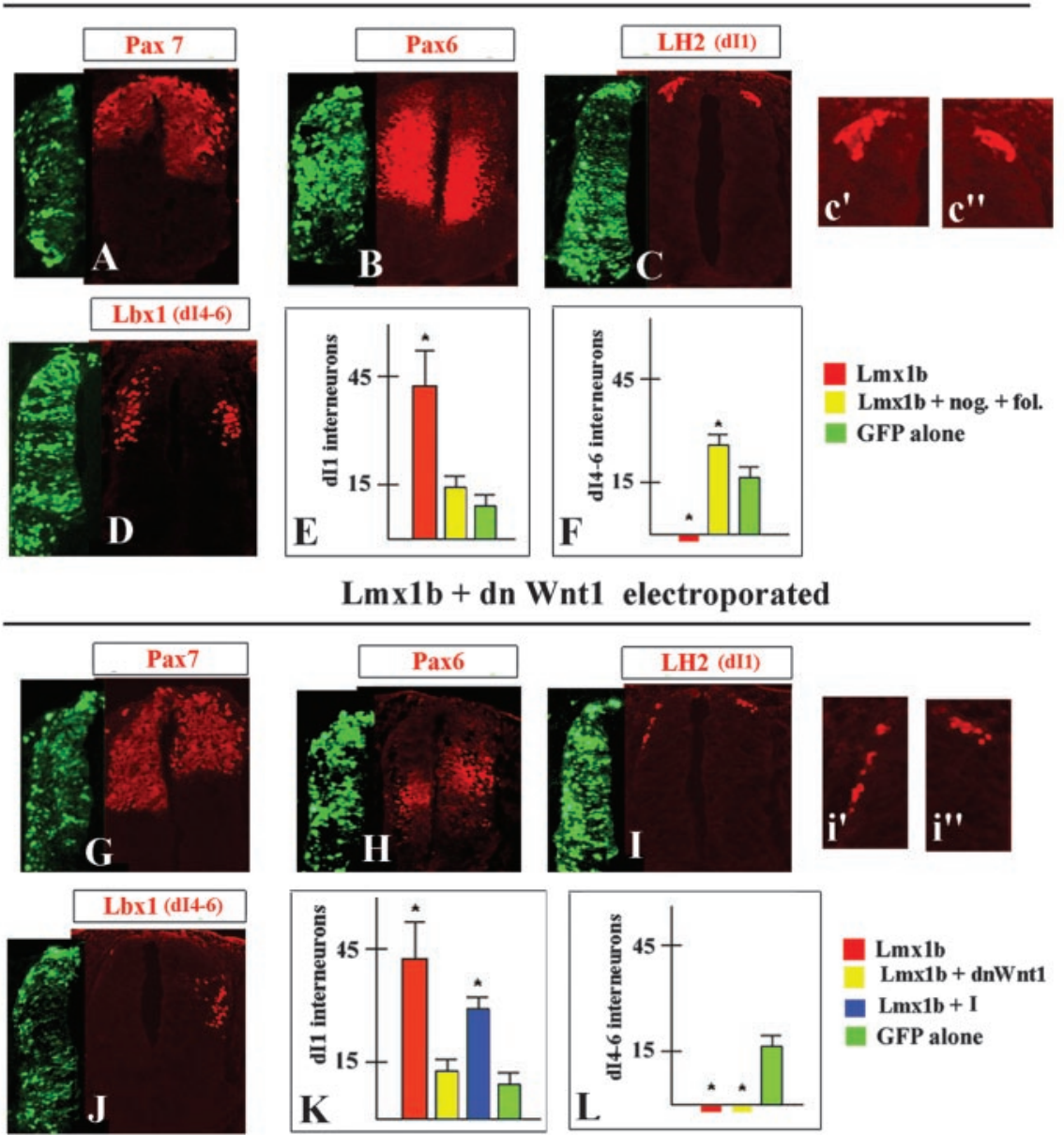

Lmx1b + noggin + follistatin + dnWnt1 electroporated
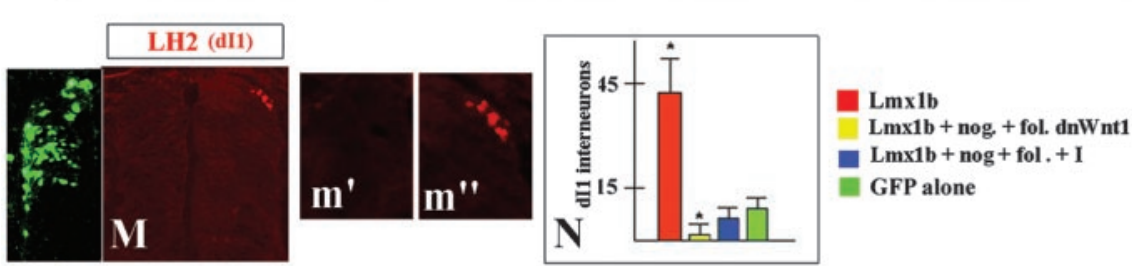

Figure 5. Blocking of $L m x 1 b$-dependent roof plate signaling by Bmps and Wnts inhibitors. Effect of overexpression of $L m x 1 b$ together with noggin (nog.) and follistatin (fol.) $(A-F), L m \times 1 b$ together with $d n W n t 1(G-L)$, and $L m \times 1 b$ together with noggin and follistatin-dnWnt1 $(M, N)$ on expression of Pax7 $(A, G), \operatorname{Pax} 6(B, H)$, and d11 $(C, I)$ and dl 4 - dl6 $(D, J)$ interneuron development. Insets show higher magnifications of selected neuronal populations. Quantitative analysis of numbers of selected classes of interneurons in neural tubes electroporated with $L m x 1 b$ together with noggin and follistatin $(E, F), L m x 1 b$ together with $d n W n t 1$ $(K, L)$, and $L m \times 1 b$ and together with noggin and follistatin - $d n W n t 1(N)$. The label I indicates cell cycle inhibitors amphidicolin and

factor that may suppress the roof plate-inducing activity of Lmx1b is Mash1. Mash1 (and its chick homolog Cash1) initiates its expression in the intermediate neural tube shortly after neural tube closure (Gowan et al., 2001) (data not shown). It has been implicated in the development of several neuronal lineages and is expressed in both dI5 and dILB progenitors before initiation of $L m \times 1 b$ expression in these differentiating neurons (Gross et al., 2002; Muller et al., 2002; Qian et al., 2002). To test whether Mash1 could suppress the roof plate-inducing activity of Lmx1b, we coelectroporated Mash1 and $L m \times 1 b$ in caudal developing chick spinal cord just before neural tube closure at stage 10. In 

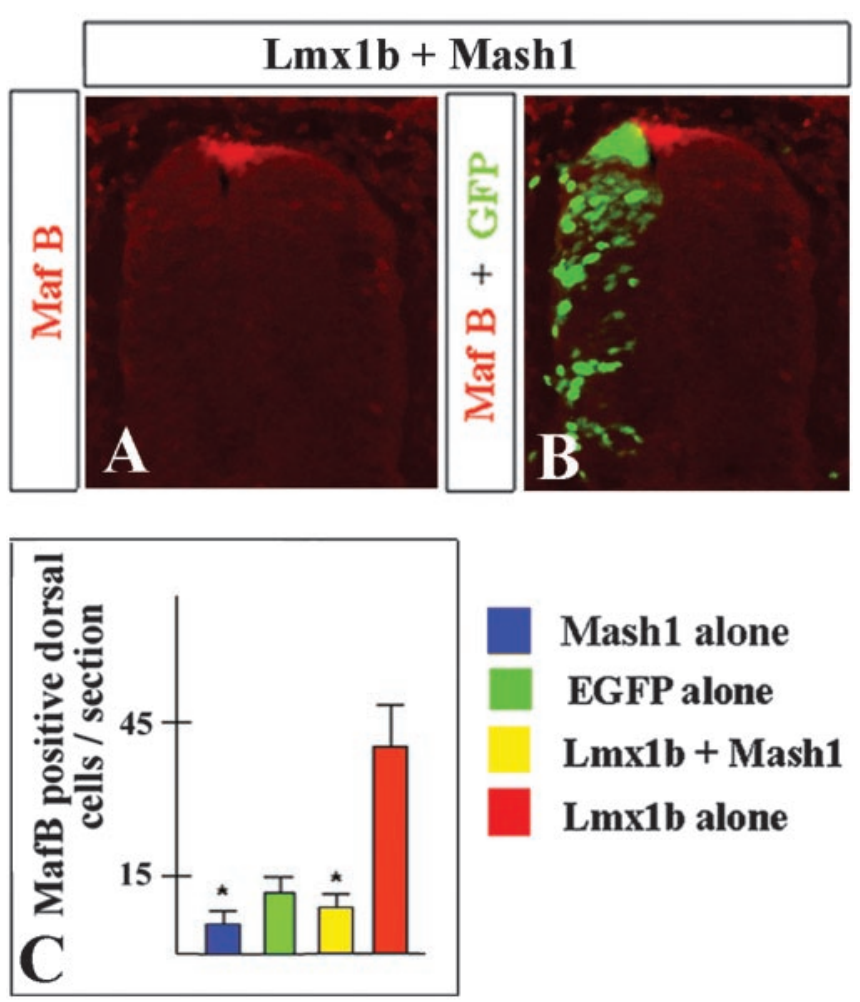

Figure 6. Mash1 suppresses roof plate-inducing activity of $L m x 1 b$. Expression of MafB (red) $(A)$ alone and together with GFP (green) $(B)$ in chick developing spinal cord coelectroporated with $L m \times 1 b$ and Mash 1, 24 h.a.e. Quantitative analysis of roof plate cells in embryos electroporated with Mash 1 alone versus EGFP alone and $L m x 1 b$ together with Mash 1 versus $L m x 1 b$ alone.

contrast to electroporation of $L m \times 1 b$ alone, coelectroporation of $L m \times 1 b$ with Mash1 failed to induce ectopic roof plate, as detected by MafB, Bmp4, or Gdf7 staining (Fig. 6 and data not shown). In addition, we observed an inhibition of endogenous roof plate formation when Mash1 alone was electroporated into chick stage 10 neural plates (Fig. $6 C$ and data not shown). Together, these data indicate that Mash1 can suppress roof plate-inducing activity of Lmxlb when overexpressed in chick developing spinal cord.

\section{Discussion}

The roof plate is an important signaling center that regulates dorsal CNS patterning during vertebrate development, yet very little is understood about the molecular pathways that drive its induction and function. In this study, we show that $L m x 1 b$ can drive ectopic roof plate formation in the developing chick spinal cord and that Bmp and Wnt signaling are major components of Lmx1-dependent roof plate signaling. This function is not conserved across vertebrate evolution because $L m \times 1 b$ is not expressed in the roof plate of mouse spinal cord. In mouse, caudal CNS roof plate formation relies entirely on Lmxla. Lmx1b acts upstream of Lmxla in the chick roof plate differentiation program and can partially rescue roof plate development in the dreher $\left(\operatorname{Lm} \times 1 a^{-/-}\right)$mouse. Although $\operatorname{Lm} \times 1 b$ is also normally expressed in dI5 and dILB interneurons in intermediate regions of the neural tube, loss-of-function or ectopic expression of $L m \times 1 b$ does not alter the specification of these neurons. We therefore conclude that Lmxib is not critically involved in dI5 and dILB interneuron specification and that roof plate induction is the primary activity of Lmxlb in early dorsal spinal cord development in chick. This activity may be suppressed by Mash1
(Cash1), normally expressed in intermediate regions of the mouse and chick neural tube.

\section{Roof plate induction mechanisms are different in chick and mouse caudal CNS}

Roof plate is an embryonic signaling center that consists of nondividing differentiated cells. In the developing vertebrate spinal cord, the roof plate forms from mitotically active progenitors at the lateral edges of the neural plate in response to Bmp signaling from adjacent ectodermal ectoderm (Lee and Jessell, 1999). Here we provide evidence that $L m x 1 b$ can induce ectopic expression of numerous roof plate markers, including Lmxla, MafB, Bmp4, Gdf7, and Wnt1, when overexpressed in stage 10 chick neural plate. Surprisingly, Lmxlb was not sufficient to withdraw progenitor cells from the cell cycle, showing that it does not control this aspect of roof plate differentiation program. Nevertheless, we consider that Lmx1b can induce "roof plate," because the induced structure non-autonomously affects the specification of adjacent neurons in the dorsal spinal cord. The results of our electroporation studies do not allow us to directly conclude that roof plate induction is the normal function of $L m \times 1 b$ in chick developing spinal cord. However, together with the fact that Lmx1b is expressed in the chick roof plate progenitors and differentiated roof plate cells and that, similar to other roof plate markers, $\mathrm{Lmx} 1 \mathrm{~b}$ expression is responsive to Bmp signaling, our data strongly suggest that $L m \times 1 b$ contributes to roof plate formation during normal chick spinal cord development.

We showed recently that $L m x 1 a$ also has roof plate-inducing activities in the chick neural plate and is regulated by Bmp signaling (Chizhikov and Millen, 2004). We conclude that, in the chick, Lmx1b and Lmxla have at least partially redundant roof plateinducing properties. However, because Lmxlb acts upstream of Lmxla, at least some of the roof plate-inducing activity of Lmx1b may be mediated by Lmxla.

In mouse caudal CNS, roof plate induction relies entirely on the action of Lmxla, because Lmxlb is never expressed in roof plate progenitors or differentiated roof plate in the mouse spinal cord. Loss of Lmxla function in the $\operatorname{dreher}\left(\operatorname{Lm} \times 1 a^{-/-}\right)$mutant mouse results in failure of roof plate development, which in turn, causes subsequent disruptions in the specification, patterning, and differentiation of adjacent dorsal sensory interneurons in the dreher spinal cord (Millonig et al., 2000). Interestingly, both chick and mouse $L m x 1 b$ can induce roof plate gene expression in the dreher spinal cord, partially rescuing the Lmxla mutant phenotype, indicating that mouse Lmxlb still has roof plate-inducing properties. This finding is also supported by the observation that chick and mouse $L m x 1 b$ have equivalent properties in chick electroporation experiments. Importantly, however, mouse $L m x 1 b$ is not expressed in roof plate progenitors or developing roof plate, despite high levels of endogenous Bmp signals from the adjacent epidermal ectoderm, levels that are sufficient to induce $\operatorname{Lmx} 1 a$. This indicates that the primary difference between chick and mouse $L m x 1 b$ is not its functional properties but rather the regulation of its expression. Cross-species and inter-loci comparative sequence analyses are likely to reveal regulatory differences contributing to this fundamental difference between mouse and chick roof plate-inductive mechanisms.

Although the roof plate-inducing activities of Lmx1a and $L m \times 1 b$ are similar, they are not entirely overlapping. In contrast to Lmxla (Chizhikov and Millen, 2004), Lmxlb overexpression cannot cause progenitor cell cycle withdrawal in either mouse explants or chick spinal cord. Thus, the cell cycle withdrawal activity is unique to Lmxla. Whether these differences are attrib- 
utable to actual sequence differences between Lmx1a and Lmx1b proteins or whether it is attributable to the requirement for different cofactors remains to be investigated.

\section{The molecular nature of Lmx1-dependent roof plate signaling} The ability of $L m \times 1 b$ and $L m \times 1 a$ to induce functional roof plate provided an opportunity to asses the molecular nature of Lmx1dependent roof plate signaling. By coexpression of $L m x 1 b$ or Lmxla together with inhibitors of Bmp and Wnt signaling in chick developing spinal cord, we showed that Bmps are major components of Lmxla/b-dependent roof plate signaling in these experimental conditions, regulating the development of many dorsal interneurons. Interestingly, loss of roof plate Bmp signaling (but not Wnt signaling) observed in the dreher mouse spinal cord is also associated with decrease of dI1 interneuron numbers (Millen et al., 2004), suggesting that Bmps may be important mediators of Lmx1 signaling not only in the chick but also in the mouse. $L m x 1 a / b$ expression also activates Wnt signaling. In contrast to widespread actions of Bmps, the action of Lmx1dependent Wnt signaling is limited to the positive regulation of dI1 interneuron development. Previous studies have identified Wntl as a mitogen for the neural cells (Megason and McMahon, 2002). We showed that the effects of cell cycle inhibitors on dI1 development in the presence of ectopic $L m \times 1 b$ are not equivalent to coexpression of $L m \times 1 b$ and Wntl inhibitors. These results indicate that Wntl plays a more direct role in patterning dI1 specification. Interestingly, overexpression of Wnt1 in chick had no significant effect on the specification of any group of dorsal or intermediate cells except dI1 interneurons, suggesting that Wnt1 is unlikely a major regulator of dorsal cell identity in chick caudal CNS. This is in contrast to mouse, in which knock-out studies identified Wnt1 and Wnt3a as major regulators of dorsal cell identity in developing neural tube (Muroyama et al., 2002). Together, this suggests that there may be differences in the role of Wnt signaling in dorsal neuronal specification between chick and mouse.

\section{Lmx1b and dI5/dILB dorsal interneuron development}

Our stage 10 neural plate electroporation data indicate that the primary function of Lmx1b in the early developing spinal cord is roof plate induction. At later stages, however, differentiating dI5 and dILB interneurons express endogenous Lmxlb in both chick and mouse without acquiring roof plate properties. By coelectroporating chick stage 15 neural tubes with $L m x 1 b$ and Mash1, which are normally expressed in intermediate neural tube including dI5 and dILB progenitors, we showed that Mash1 can inhibit the roof plate-inducing activity of Lmx1b. Thus, Mash1 (Cash1) may be one factor that prevents $\mathrm{dI} 5$ and dILB progenitors from adopting roof plate properties. Analysis of $\mathrm{Mash}^{-/-}$mice is required to further support this hypothesis.

Interestingly, our loss-of-function studies in mouse revealed no role for Lmx1b in dI5 and dILB interneuron specification. This conclusion was further supported by gain-of-function experiments, performed on stage 15 chick developing spinal cord, at the time of initiation of expression of endogenous $L m \times 1 b$ in dI5 interneurons. These data show that, at this stage, exogenous Lmxlb can neither induce additional dI5 interneurons nor block normal specification of other dorsal interneurons. This is in contrast to other $L m x 1 b$-expressing neuronal populations in the CNS in which $L m \times 1 b$ regulates cell fate [e.g., dopaminergic and serotonergic neurons (Smidt et al., 2000; Ding et al., 2003)]. Additional analysis of dI5/dILB neuronal migration and their axon development is required to determine whether $L m x 1 b$ is involved in later steps of development of dI5 and dILB neurons.

\section{References}

Adams KA, Maida JM, Golden JA, Riddle RD (2000) The transcription factor Lmxlb maintains Wntl expression within the isthmic organizer. Development 127:1857-1867.

Akamatsu W, Okano HJ, Osumi N, Inoue T, Nakamura S, Sakakibara S, Miura M, Matsuo N, Darnell RB, Okano H (1999) Mammalian ELAVlike neuronal RNA-binding proteins $\mathrm{HuB}$ and $\mathrm{HuC}$ promote neuronal development in both the central and the peripheral nervous systems. Proc Natl Acad Sci USA 96:9885-9890.

Butler SJ, Dodd J (2003) A role for BMP heterodimers in roof platemediated repulsion of commissural axons. Neuron 38:389-401.

Chen H, Lun Y, Ovchinnikov D, Kokubo H, Oberg KC, Pepicelli CV, Gan L, Lee B, Johnson RL (1998) Limb and kidney defects in Lmxlb mutant mice suggest an involvement of LMX1B in human nail patella syndrome. Nat Genet 19:51-55.

Chizhikov VV, Millen KJ (2004) Control of roof plate formation by Lmxla in the developing spinal cord. Development 131:2693-2705.

Ding YQ, Marklund U, Yuan W, Yin J, Wegman L, Ericson J, Deneris E, Johnson RL, Chen ZF (2003) Lmxlb is essential for the development of serotonergic neurons. Nat Neurosci 6:933-938.

Dreyer SD, Zhou G, Baldini A, Winterpacht A, Zabel B, Cole W, Johnson RL, Lee B (1998) Mutations in LMX1B cause abnormal skeletal patterning and renal dysplasia in nail patella syndrome. Nat Genet 19:47-50.

Ericson J, Rashbass P, Schedl A, Brenner-Morton S, Kawakami A, van Heyningen V, Jessell TM, Briscoe J (1997) Pax6 controls progenitor cell identity and neuronal fate in response to graded Shh signaling. Cell 90:169-180.

Failli V, Bachy I, Retaux S (2002) Expression of the LIM-homeodomain gene Lmxla (dreher) during development of the mouse nervous system. Mech Dev 118:225-228.

Fedtsova N, Turner EE (1997) Inhibitory effects of ventral signals on the development of Brn-3.0-expressing neurons in the dorsal spinal cord. Dev Biol 190:18-31.

Garcia-Castro MI, Marcelle C, Bronner-Fraser M (2002) Ectodermal Wnt function as a neural crest inducer. Science 297:848-851.

Gowan K, Helms AW, Hunsaker TL, Collisson T, Ebert PJ, Odom R, Johnson JE (2001) Crossinhibitory activities of Ngn1 and Math1 allow specification of distinct dorsal interneurons. Neuron 31:219-232.

Gross MK, Dottori M, Goulding M (2002) Lbxl specifies somatosensory association interneurons in the dorsal spinal cord. Neuron 34:535-549.

Hamburger V, Hamilton HL (1951) A series of normal stages in the development of the chick embryo. J Morphol 88:49-92.

Helms AW, Johnson JE (1998) Progenitors of dorsal commissural interneurons are defined by MATH1 expression. Development 125:919-928.

Helms AW, Johnson JE (2003) Specification of dorsal spinal cord interneurons. Curr Opin Neurobiol 13:42-49.

Hobert O, Westphal H (2000) Functions of LIM-homeobox genes. Trends Genet 16:75-83.

Kishimoto KN, Watanabe Y, Nakamura H, Kokubun S (2002) Ectopic bone formation by electroporatic transfer of bone morphogenetic protein- 4 gene. Bone 31:340-347.

Lee KJ, Jessell TM (1999) The specification of dorsal cell fates in the vertebrate central nervous system. Annu Rev Neurosci 22:261-294.

Lee KJ, Mendelsohn M, Jessell TM (1998) Neuronal patterning by BMPs: a requirement for GDF7 in the generation of a discrete class of commissural interneurons in the mouse spinal cord. Genes Dev 12:3394-3407.

Liem KF, Tremml G, Roelink H, Jessell TM (1995) Dorsal differentiation of neural plate cells induced by Bmp-mediated signals from epidermal ectoderm. Cell 82:969-979.

Liem KF, Tremml G, Jessell TM (1997) A role for the roof plate and its resident TGFbeta-related proteins in neuronal patterning in the dorsal spinal cord. Cell 91:127-138.

Liem KF, Jessell TM, Briscoe J (2000) Regulation of the neural patterning activity of sonic hedgehog by secreted BMP inhibitors expressed by notochord and somites. Development 127:4855-4866.

Liu Y, Helms AW, Johnson JE (2004) Distinct activities of Msx1 and Msx3 in dorsal neural tube development. Development 131:1017-1028.

Matsunaga E, Katahira T, Nakamura H (2002) Role of Lmxlb and Wnt1 in 
mesencephalon and metencephalon development. Development 129:5269-5277.

Megason SG, McMahon AP (2002) A mitogen gradient of dorsal midline Wnts organizes growth in the CNS. Development 129:2087-2098.

Millen KJ, MillonigJH, Matten ME (2004) Roof plate and dorsal spinal cord dI1 interneuron development in the dreher mutant mouse. Dev Biol, in press.

Millonig JH, Millen KJ, Hatten ME (2000) The mouse Dreher gene Lmxla controls formation of the roof plate in the vertebrate CNS. Nature 403: $764-769$.

Muller T, Brohmann H, Pierani A, Heppenstall PA, Lewin GR, Jessell TM, Birchmeier C (2002) The homeodomain factor lbxl distinguishes two major programs of neuronal differentiation in the dorsal spinal cord. Neuron 34:551-562.

Muroyama Y, Fujihara M, Ikeya M, Kondoh H, Takada S (2002) Wnt signaling plays an essential role in neuronal specification of the dorsal spinal cord. Genes Dev 16:548-553.

Pouponnot C, Nishizawa M, Calothy G, Pierani A (1995) Transcriptional stimulation of the retina-specific QR1 gene upon growth arrest involves a Maf-related protein. Mol Cell Biol 15:5563-5575.
Qian Y, Shirasawa S, Chen CL, Cheng L, Ma Q (2002) Proper development of relay somatic sensory neurons and D2/D4 interneurons requires homeobox genes Rnx/Tlx-3 and Tlx-1. Genes Dev 16:1220-1233.

Riddle RD, Ensini M, Nelson C, Tsuchida T, Jessell TM, Tabin C (1995) Induction of the LIM homeobox gene Lmxl by WNT7a establishes dorsoventral pattern in the vertebrate limb. Cell 83:631-640.

Smidt MP, Asbreuk CH, Cox JJ, Chen H, Johnson RL, Burbach JP (2000) A second independent pathway for development of mesencephalic dopaminergic neurons requires Lmx1b. Nat Neurosci 3:337-341.

Timmer JR, Wang C, Niswander L (2002) BMP signaling patterns the dorsal and intermediate neural tube via regulation of homeobox and helix-loophelix transcription factors. Development 129:2459-2472.

Vogel A, Rodriguez C, Warnken W, Izpisua Belmonte JC (1995) Dorsal cell fate specified by chick Lmx1 during vertebrate limb development. Nature 378:716-720.

Yuan S, Schoenwolf GC (1999) The spatial and temporal pattern of C-Lmx1 expression in the neuroectoderm during chick neurulation. Mech Dev $88: 243-247$. 